University of New Hampshire

University of New Hampshire Scholars' Repository New Hampshire Agricultural Experiment Station New Hampshire Agricultural Experiment Station
Publications

$1-13-2016$

\title{
Genetic variation within and among asexual populations of Porphyra umbilicalis Kützing (Bangiales, Rhodophyta) in the Gulf of Maine, USA
}

\author{
Renee L. Eriksen \\ University of New Hampshire, Durham \\ Lindsay A. Green \\ University of New Hampshire, Durham \\ Anita S. Klein \\ University of New Hampshire, Durham, Anita.Klein@unh.edu
}

Follow this and additional works at: https://scholars.unh.edu/nhaes

Comments

This is an article published by De Gruyter in Botanica Marina in 2016, available online: https://dx.doi.org/10.1515/

bot-2015-0017

\section{Recommended Citation}

Renée L. Eriksen, Lindsay A. Green and Anita S. Klein. Genetic variation within and among asexual populations of Porphyra umbilicalis Kützing (Bangiales, Rhodophyta) in the Gulf of Maine, USA. Botanica Marina 2016; 59(1): 1-12. https://dx.doi.org/10.1515/bot-2015-0017

This Article is brought to you for free and open access by the New Hampshire Agricultural Experiment Station at University of New Hampshire Scholars' Repository. It has been accepted for inclusion in New Hampshire Agricultural Experiment Station Publications by an authorized administrator of University of New Hampshire Scholars' Repository. For more information, please contact Scholarly.Communication@unh.edu. 
Renée L. Eriksen*, Lindsay A. Green and Anita S. Klein

\section{Genetic variation within and among asexual populations of Porphyra umbilicalis Kützing (Bangiales, Rhodophyta) in the Gulf of Maine, USA}

DOI 10.1515/bot-2015-0017

Received 16 February, 2015; accepted 8 December, 2015; online first 13 January, 2016

\begin{abstract}
The intertidal marine red alga Porphyra umbilicalis reproduces asexually in the Northwest Atlantic. We looked for population substructure among typical opencoastal and atypical estuarine habitats in seven asexual populations of $P$. umbilicalis from Maine to New Hampshire using eight expressed sequence tag-simple sequence repeats (EST-SSR) or microsatellite loci. Six genotypes were identified, four of which may represent recombinant genotypes from a recombination event that took place locally, or that took place prior to introduction to the Northwest Atlantic. Genotypic diversity was lowest in a population from Wiscasset, Maine, which inhabits an atypical habitat high in the intertidal zone of a bridge piling in an estuarine tidal rapid. Genotypic diversity was highest in the southernmost populations from New Hampshire; we identified two genotypes that were unique to the southernmost populations, and probably represent the most derived genotypes. We looked at genetic distances among populations in similar habitats, and found that populations were more closely related to their closest neighboring population than to a population in a similar habitat. We show that genotypic diversity within P. umbilicalis populations in the Gulf of Maine is relatively high and thus fits a model of high steady-state variation within asexual populations.
\end{abstract}

Keywords: asexual evolution; EST-SSR; isolation-by-distance; population genetics.

*Corresponding author: Renée L. Eriksen, Department of Biological Sciences, University of New Hampshire, Durham, NH 03824, USA; and United States Department of Agriculture - Agricultural Research Service, 1636 East Alisal Street, Salinas, CA 93905, USA,

e-mail: renee.eriksen@gmail.com

Lindsay A. Green: Department of Biological Sciences, University of New Hampshire, Durham, NH 03824, USA; and Department of Biological Sciences, University of Rhode Island, Kingston, RI 02881, USA

Anita S. Klein: Department of Biological Sciences, University of New Hampshire, Durham, NH 03824, USA

\section{Introduction}

The marine red alga Porphyra umbilicalis Kützing (Bangiales, Rhodophyta) is found in the intertidal region on rocky substrata along the North American and European coasts of the North Atlantic (Brodie et al. 2008). The species and some of its close relatives are well known for some remarkable physiological traits, which make them particularly tolerant of a number of abiotic stress factors. Newly developed genomic resources (Chan et al. 2012) potentially make $P$. umbilicalis a useful model in understanding stress tolerance (Blouin et al. 2011). It has also been suggested as a potential crop in integrated multitrophic aquaculture systems (Blouin et al. 2007).

The species' geographic distribution was probably strongly affected by the previous glacial period, when glacial scouring is theorized to have extirpated obligate rocky shore organisms from Northwest (NW) Atlantic coasts (Ingolfsson 1992). Almost all obligate rocky shore invertebrate species that presently inhabit these coasts have a close relative that inhabits Northeast (NE) Atlantic rocky coasts. These NW Atlantic rocky coast species are assumed to be the descendants of NE Atlantic species that were introduced from glacial refugia in the NE Atlantic via post-glacial trans-Atlantic currents that allowed colonization of Iceland, Greenland, and then south along the coast of North America (Ingolfsson 1992), where prevailing currents would have allowed colonization of the Gulf of Maine from a north to south direction. To support this theory in marine macroalgae, Teasdale and Klein (2010) used ribosomal introns and the ITS1-5.8S - ITS2 region to show that populations of $P$. umbilicalis from the NW Atlantic were probably derived from European populations after the last glacial maximum.

Populations of $P$. umbilicalis in Great Britain reproduce sexually and asexually (Brodie and Irvine 2003) but, for reasons that are not yet known, NW Atlantic populations appear to reproduce only asexually via neutral spores (Blouin et al. 2007, Blouin 2010, Blouin and Brawley 2012). Neutral spores are produced via mitosis of somatic cells around the edge of the blade, and grow via mitosis directly 
into new blades (Nelson et al. 1999). The dominant life history phase on both sides of the Atlantic is a haploid blade; the diploid conchocelis stage of the life cycle is apparently bypassed entirely in NW Atlantic populations.

In this study, we assayed eight loci mined from the P. umbilicalis Expressed Sequence Tag (EST) database (Chan et al. 2012) in five typical open-coastal and two atypical estuarine tidal-rapid populations of $P$. umbilicalis from New Hampshire and Maine, USA (Table 1) to look for genetic variation in simple sequence repeats (SSRs) or microsatellites. Microsatellites are hypervariable regions of the genome (Chakraborty et al. 1997) that allow us to discriminate differences among populations within the same species. As $P$. umbilicalis reproduces asexually in the NW Atlantic, sexual recombination within the genome does not occur, and thus the panels of microsatellite loci assayed here are presented as linked alleles comprising a genotype or lineage, rather than as individual, freelyrecombining, neutral alleles as microsatellite data are typically presented. Our goal was to look for patterns in genetic variation among populations in open-coastal and estuarine tidal rapid habitats at a relatively fine scale (the Gulf of Maine) to elucidate the factors that dictate population structure in asexual populations of P. umbilicalis from the NW Atlantic.

\section{Materials and methods}

Tissue for population genetic surveys was collected throughout the year at low tide from seven sites in New Hampshire (NH) and Maine (ME; Table 1). For all sites but Dover Point and Wiscasset, we selected a random position along the rocky shore during low tide, and traveled both parallel and perpendicular to the tide line collecting 3-5 entire "individuals" (defined here as thalli connected by the same holdfast) from rocks within a 1-m radius of a random stopping point. At Dover Point and Wiscasset, the populations inhabit estuarine locations in the high-intertidal region of concrete bridge pilings, where tidal flow and a narrow constriction of the waterway combine to create tidal rapids. Maximum tidal flow at Dover Point has been measured at 5.2 knots (Mathieson et al.1981). Individuals were taken directly from the bridge piling during low tide while wading (Dover Point) or in a boat (Wiscasset), and attempts were made to take similar numbers of "individuals" from each position on the piling.

Genomic DNA was extracted using a modification of a protocol developed by Page(2010) substituting 1.8 M sodium chloride and a 1-h ethanol precipitation after optimization of the procedure. DNA concentration was assessed using a NanoDrop 2000c spectrophotometer (Thermo Scientific, Wilmington, DE, USA). Non-reproductive accessions within the foliose Bangiales group are hard to distinguish by morphology alone. Therefore, in order to confirm species identification, a subset of samples from open-coastal populations and all samples from Dover Point and Wiscasset were first assayed by restriction fragment length polymorphism (RFLP) assays according to Teasdale et al. (2002).

The Porphyra umbilicalis nuclear nonredundant EST database assembled in September 2010 was downloaded from the Porphyra Research Coordination Network (RCN) website (http://dbdata.rutgers.edu/nori/). Contigs containing 10 or more two- or three-base repeats and five or more 4-base repeats were identified by MISA (Thiel et al. 2003). Primer3 (Rozen and Skaletsky 2000) was used to design primers in sequences containing sufficient flanking data. Amplification of each region was conducted in $25 \mu \mathrm{l}$ reactions containing about 25-125 ng DNA, 1x OneTaq ${ }^{\mathrm{TM}}$ GC Reaction Buffer (New England BioLabs, Ipswich, MA, USA), 15\% OneTa ${ }^{\mathrm{TM}}$ GC Enhancer containing 25\% DMSO (New England BioLabs), $200 \mu \mathrm{M}$ dNTPs, $0.8 \% \mathrm{BSA}, 0.2 \mu \mathrm{M}$ of each the forward and reverse primers, and OneTaq ${ }^{\mathrm{TM}}$ Hot Start DNA Polymerase according to the manufacturer's instructions (New England BioLabs). PCR conditions involved an initial 5-min hot start at $95^{\circ} \mathrm{C}$, a denaturation at $95^{\circ} \mathrm{C}$ for $30 \mathrm{~s}$, a primer-specific annealing temperature (Table 2 ) that decreased $2^{\circ} \mathrm{C}$ every second

Table 1: Location, season and number of samples collected from Porphyra umbilicalis populations in typical open-coastal and atypical estuarine tidal rapid habitats in Maine (ME) and New Hampshire (NH), USA.

\begin{tabular}{llclll}
\hline Habitat & Population & No. samples & Season of collection & Town, state & GPS coordinates \\
\hline Open-coastal & Quoddy Head & 47 & Winter & Lubec, ME & $44^{\circ} 48^{\prime} 49.2^{\prime \prime} \mathrm{N} 66^{\circ} 57^{\prime} 06.9^{\prime \prime} \mathrm{W}$ \\
& Schoodic Point & 23 & Winter & Winter Harbor, ME & $44^{\circ} 20^{\prime} 11.9^{\prime \prime} \mathrm{N} 68^{\circ} 03^{\prime} 22.8^{\prime \prime} \mathrm{W}$ \\
& Reid State Park & 34 & Winter & Georgetown, ME & $43^{\circ} 46^{\prime} 29.0^{\prime \prime} \mathrm{N} 69^{\circ} 43^{\prime} 53.7^{\prime \prime} \mathrm{W}$ \\
& Nubble Light & 25 & Summer & York, ME & $43^{\circ} 09^{\prime} 57.9^{\prime \prime} \mathrm{N} 70^{\circ} 35^{\prime} 34.4^{\prime \prime} \mathrm{W}$ \\
& Fort Stark & 39 & Summer & New Castle, NH & $43^{\circ} 03^{\prime} 28.3^{\prime \prime} \mathrm{N} 70^{\circ} 42^{\prime} 41.7^{\prime \prime} \mathrm{W}$ \\
Estuarine tidal & Wiscasset & 44 & Late fall & Wiscasset, ME & $43^{\circ} 58^{\prime} 22.3^{\prime \prime} \mathrm{N} 69^{\circ} 40^{\prime} 24.2^{\prime \prime} \mathrm{W}$ \\
Rapids & Dover Point & 9 & Multiple seasons & Dover Point, NH & $43^{\circ} 07^{\prime} 11.5^{\prime \prime} \mathrm{N} 70^{\circ} 49^{\prime} 38.5^{\prime \prime} \mathrm{W}$ \\
\hline
\end{tabular}


Table 2: Primers used to amplify expressed sequence tag - simple sequence repeat (EST-SSR) regions in Porphyra umbilicalis.

\begin{tabular}{llrrlll}
\hline Primer name & Motif & $\begin{array}{r}\text { Amplicon } \\
\text { length }\end{array}$ & $\begin{array}{r}\text { Annealing } \\
\text { temperature }\end{array}$ & Left primer & Right Primer & Contig ID \\
\hline 399 & TGC & 252 & 52 & AGCACCTTTTACCGGTGTTG & GGGCTTCCATGAAGATGATG & Contig399 \\
1366 & GGC & $224,245,252$ & 52 & ACAGCTACTTTGGCGGACAG & CGGATTGGTTATAGCCTTGC & Contig1366 \\
2219 & TTG & 276 & 54 & ACGGCGACACGAAGTAGC & GTGGGAATGGGACAACAAC & NBisotig02219 \\
4157 & CCG & 291 & 52 & GTCCAAGGCGTCGGTTAG & CGAAAAAGAATGGGACAAG & Contig4157 \\
4232 & CGG & 258 & 54 & GACTTGACGGGTGCCTTG & GACCTGCACCCGAGATTG & NBisotig04232 \\
5870 & GAC & 193,211 & 54 & GACCTGCTGAATGACGACAA & TCTTGATGACTTGCCGTG & NBisotig05870 \\
6274 & GCT & 271 & 54 & CCCCTCACTCCTCGTCATAC & GGGGATAGCTCGTCTTCCTC & NBisotig06274 \\
10210 & CAG & 268 & 52 & GAGTCACTGATGGCGATGG & GGTGCATTGACTGCAGGTG & NBisotig10210 \\
\hline
\end{tabular}

The primer name or locus name is based on the contig ID from an assembly downloaded in September, 2010. The motif is the 3-base repeated sequence factor within the repeat region according to bioinformatics analysis, and the amplicon length is the size of the PCR amplicon(s) for each locus. The starting annealing temperature for touchdown PCR protocols is given; the annealing temperature decreased $2^{\circ} \mathrm{C}$ every second cycle from this starting temperature. Left and right or forward and reverse primer sequences are also given.

cycle ("touchdown" protocol), followed by an extension step at $68^{\circ} \mathrm{C}$ for $45 \mathrm{~s}$. These steps were repeated for 45 cycles, then followed by an additional denaturation period at $95^{\circ} \mathrm{C}$ for $30 \mathrm{~s}$, an annealing period at $10^{\circ} \mathrm{C}$ lower than the previous starting annealing temperature for $30 \mathrm{~s}$, and an extension period at $68^{\circ} \mathrm{C}$ for $45 \mathrm{~s}$ repeated five times, and a final extension cycle at $68^{\circ} \mathrm{C}$ for $10 \mathrm{~min}$. Genotyping was conducted at the Hubbard Center for Genomic Studies at the University of New Hampshire on an ABI 3100 Genetic Analyzer (ThermoFisher Scientific, Waltham, MA, USA). Microsatellite peaks were analysed in Peak Scanner (v. 1) software (ThermoFisher Scientific, Waltham, MA, USA), and all peaks were confirmed visually. A subset of samples comprising about $10 \%$ of the total were re-run to confirm genotypes.

A total of 221 individuals were assayed for eight expressed sequence tag-simple sequence repeats (ESTSSR) loci (Table 2). To eliminate doubt about species identity, a subset of samples of each genotype (1-3 individuals of each genotype) identified by EST-SSRs from each population (subset $=48$ individuals) was post-hoc sequenced for the 298 base pair $r b c \mathrm{~L}-r b c \mathrm{~S}$ intergenic spacer using primers RBCL5RC and RBCSPC according to MolsMortensen et al. (2012) and Bray et al. (2006). Sequencing was conducted at the Hubbard Center for Genomic Studies on an ABI 3100 Genetic Analyzer, and sequences were edited and screened for polymorphisms in the software Lasergene (DNASTAR, Madison, WI, USA).

Nei's unbiased genetic distances among populations were calculated using genotype frequencies in GenAlEx v. 6.41 (Peakall and Smouse 2006, Peakall and Smouse 2012), and principal components analyses (PCA) were performed using Nei's unbiased genetic distances. Mantel tests for isolation-by-distance were performed in R v. 3.2.1 (R Core Team 2015) using the ade4 package (Chessel et al. 2004,
Dray and Dufour 2007, Dray et al. 2007) based on Nei's unbiased genetic distances and geographic distances, which were calculated as the shortest distance between GPS coordinates (Table 1) using the haversine formula. Because neutral spores cannot disperse via land routes, the distance between any open-coastal population and any estuarine population was calculated as the distance between the open-coastal population in question and the nearest open-coastal population to the estuarine population, plus the distance between the estuarine population and its nearest open-coastal population (e.g. the distance between Schoodic Point and Dover Point was calculated as the distance between Schoodic Point and Fort Stark plus the distance between Fort Stark and Dover Point). Mantel p-values were calculated based on 10,000 replicates. Analysis of molecular variance (AMOVA) was performed in Arlequin v. 3.1.1 (Excoffier et al. 1992, Excoffier et al. 2005, Excoffier and Lischer 2010).

\section{Results}

All samples from open-coastal habitats and most samples from estuarine habitats were identified as Porphyra umbilicalis by RFLP patterns. The red alga Porphyra purpurea was somewhat more common than P. umbilicalis at Dover Point, and samples with the P. purpurea RFLP pattern were eliminated from the collection. A subset of remaining P. umbilicalis samples was sequenced for the $298 \mathrm{bp}$ $r b c \mathrm{~L}-r b c \mathrm{~S}$ intergenic spacer after EST-SSR screenings, and no polymorphisms were detected in this sequence (data not shown). Sequences were $100 \%$ identical to a $P$. umbilicalis plastid partial genome (GenBank accession JQ408795.1; Smith et al. 2012), and several P. umbilicalis voucher samples (ex. GenBank accession JN787114.1). 
Five of the eight EST-SSR loci assayed were consistently monomorphic across the populations surveyed (loci 1366, 399, 10210, 6274, 4232; Table 2). The remaining three loci were polymorphic across one or more populations. Microsatellite peaks were highly reproducible among technical replicates. The number of alleles per locus ranged from two $(2179,05870)$ to three (02219; Table 2).

We detected six different genotypes consisting of linked alleles among all populations, though all six genotypes were never observed in a single population. All populations contained a "universal" genotype (genotype 2) that was present in varying proportions in all populations. On average, this "universal" genotype made up $26 \%$ of the population, and ranged among all populations from $7 \%$ (Fort Stark) to 40\% (Dover Point; Figure 1).

Four genotypes (genotypes 1, 2, 4, 5; Table 3) contained alleles that were not always completely linked and appear, on the surface, to be recombinant genotypes. Alleles A and B from locus 2179 were found with either allele A or B from locus 2219 in these genotypes. These genotypes (genotypes 1, 2, 4, and 5; Table 3) were the most common genotypes among the populations sampled. The frequency of each of these genotypes varied widely among populations (Figure 1), and no population contained all four of these genotypes.

The remaining two genotypes, genotypes 3 and 6, are closely related to genotype 1 (Figure 2), differing by only one allele at different loci (Table 3). Both are unique to southern populations in New Hampshire (Dover Point and Fort Stark). Genotype 1, however, has not been sampled from either of these populations. Genotype 6 is the only genotype unique to a single population and is found only at Fort Stark, where it makes up about $10 \%$ of the population sampled (Figure 1). Due to the presence of these two additional genotypes, New Hampshire populations were more genotypically diverse than populations from the coast of Maine.

In addition to being the most genotypically diverse, populations from New Hampshire were more closely related to each other than to populations from northern Maine based on Nei's unbiased genetic distances calculated using genotype frequencies (Figure 3). Fort Stark and Dover Point populations shared some of the lowest genetic distances measurements (Table 4), along with Dover Point and Nubble Light. Despite this example, however, there was no correlation between Nei's unbiased genetic distances and geographic distance among all populations sampled in the Gulf of Maine (Mantel test: $\mathrm{R}^{2}=-0.10, \mathrm{p}=0.60$ ).

The AMOVA is a method analogous to analysis of variance, and it attempts to describe the partitioning of genetic variation within and among populations (Excoffier et al. 1992). For P. umbilicalis populations in the Gulf of Maine, however, AMOVA analysis found that variation within population accounted for $58 \%$ of the total variation, and $42 \%$ of the total variation was partitioned among populations $\left(\Phi_{\mathrm{PT}}=0.42, \mathrm{p}<0.001\right)$.

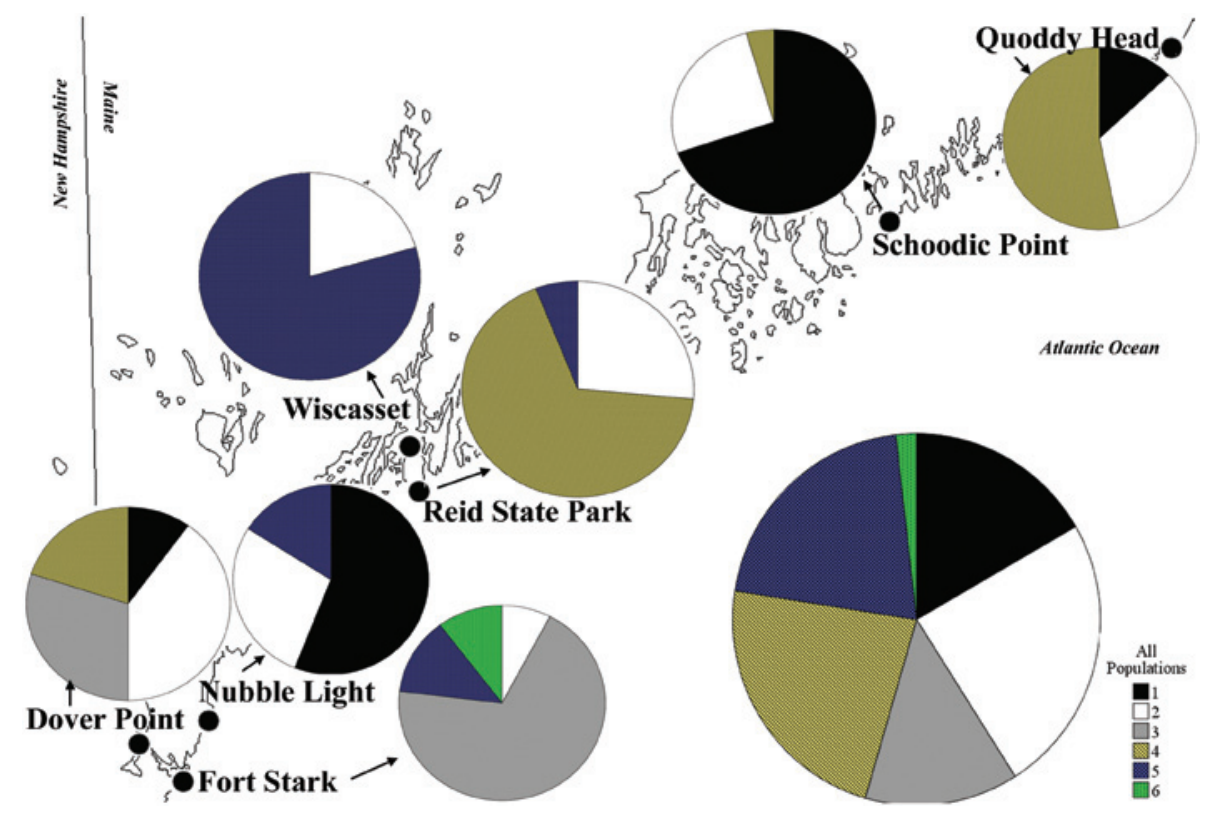

Figure 1: Collection localities of Porphyra umbilicalis populations in New Hampshire and Maine, USA. Pie charts show frequencies of genotypes in each population collection. The sum of genotype frequencies among all populations is also given. 
Table 3: Genotypes and the alleles at the three polymorphic loci $(2179,2219,5870)$ for Porphyra umbilicalis populations in the Gulf of Maine, USA.

\begin{tabular}{llll}
\hline Genotype & $\mathbf{2 1 7 9}$ & $\mathbf{2 2 1 9}$ & $\mathbf{5 8 7 0}$ \\
\hline 1 & $\mathrm{~A}_{151}$ & $\mathrm{~A}_{224}$ & $\mathrm{~A}_{193}$ \\
2 & $\mathrm{~A}_{151}$ & $\mathrm{~B}_{245}$ & $\mathrm{~A}_{193}$ \\
3 & $\mathrm{~A}_{151}$ & $\mathrm{C}_{252}$ & $\mathrm{~A}_{193}$ \\
4 & $\mathrm{~B}_{160}$ & $\mathrm{~A}_{224}$ & $\mathrm{~A}_{193}$ \\
5 & $\mathrm{~B}_{160}$ & $\mathrm{~B}_{245}$ & $\mathrm{~A}_{193}$ \\
6 & $\mathrm{~A}_{151}$ & $\mathrm{~A}_{224}$ & $\mathrm{~B}_{211}$ \\
\hline
\end{tabular}

Letters refer to the allele, while the subscripts represent the amplicon fragment sizes.

\section{Discussion}

As the genomic resources for Porphyra umbilicalis grow, the species will become useful as a model for abiotic stress tolerance in plants, and potentially as an important partner in integrated multitrophic aquaculture systems. However, it is important to understand when considering P. umbilicalis in these models that, although the species reproduces asexually in the NW Atlantic, populations even in a relatively small geographic region such as the Gulf of Maine are not depauperate of genetic variation, and we cannot assume that any two individuals are genetically identical.
A number of studies have recently emerged attempting to model and understand the factors that affect the rate of evolution in asexual organisms (Thompson et al. 2006, Desai and Fisher 2007, Desai et al. 2007, Weissman et al. 2009, Desai and Fisher 2011, Lang et al. 2011, Good et al. 2012, Kryazhimskiy et al. 2012), and one important insight that has emerged is the importance of linked mutations within a single lineage or genotype that occur in succession (Desai et al. 2007, Lang et al. 2011). These studies have found that, when the mutation rate is high, mutations are common, and a new genetic lineage arises every time there is a new mutation. Each new lineage contains the mutations of its parent lineage as well as its own new mutations. Every new mutation could be beneficial, detrimental, neutral, or nearly neutral, and lacking recombination to mix and match beneficial mutations and to eliminate deleterious mutations, the fitness of a single lineage is the sum of the accumulated mutations within that lineage. The fitness of the population is the sum of the fitness values of all the lineages, which can vary considerably within the population. Genetic variation within the population grows with the birth of each new lineage, and the variation in fitness within the population thus also increases. Selection can eliminate whole lineages with very low fitness, eventually creating a steady-state balance between mutation (which increases the variation

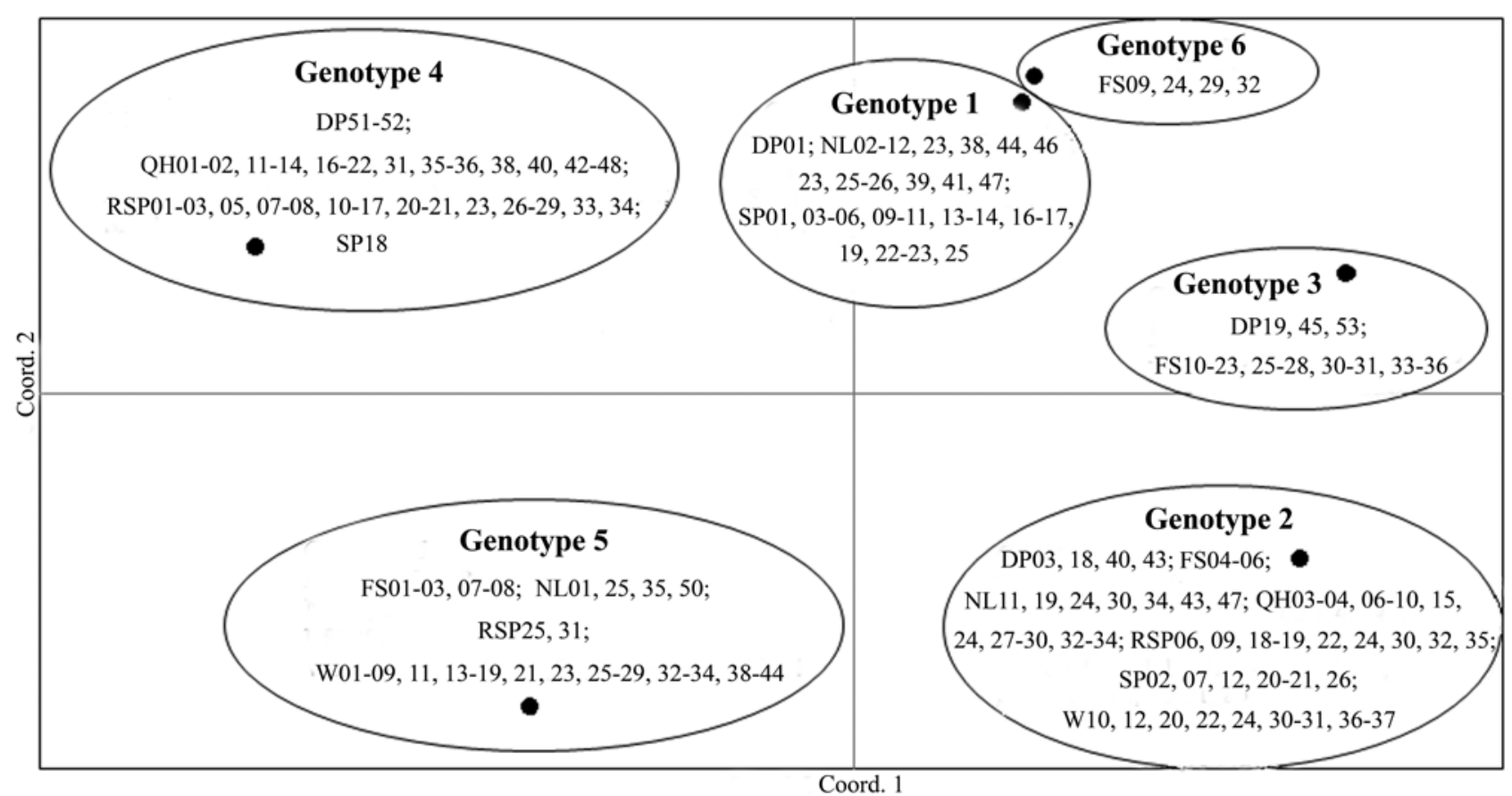

Figure 2: Principal Components Analysis based on Nei's genetic distances among Porphyra umbilicalis genotypes. The genotypes as well as the individuals assayed with the genotype are given (DP=Dover Point, FS=Fort Stark, NL=Nubble Light, QH=Quoddy Head, RSP=Reid State Park, and W=Wiscasset). 


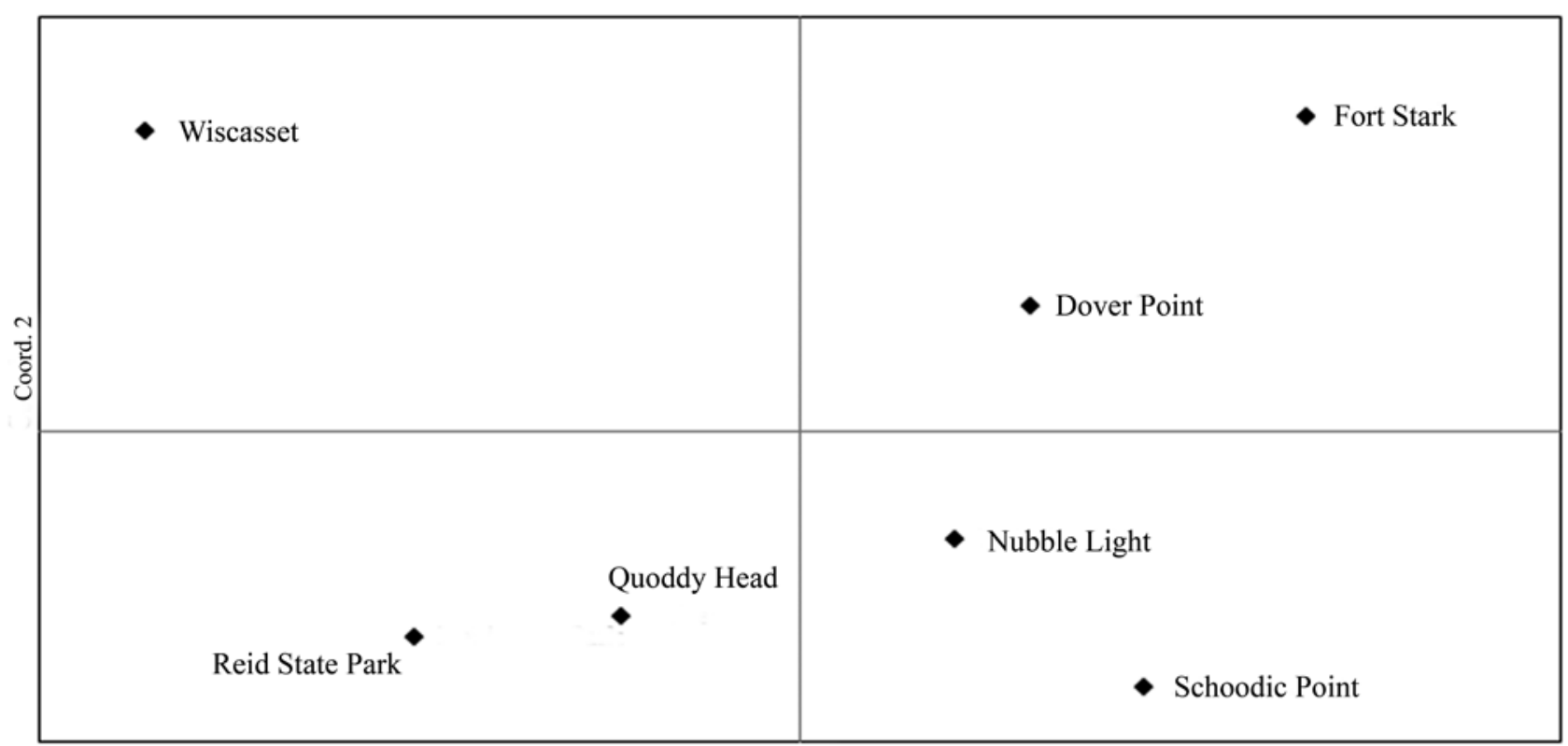

Coord. 1

Figure 3: Principal components analysis based on Nei's unbiased genetic distances among populations of Porphyra umbilicalis in the Gulf of Maine, USA.

Table 4: Pairwise comparisons using Nei's unbiased genetic distances of Porphyra umbilicalis populations in the Gulf of Maine calculated using genotype frequencies.

\begin{tabular}{|c|c|c|c|c|c|c|}
\hline & Dover Point & Fort Stark & Quoddy Head & Nubble Light & Reid State Park & Schoodic Point \\
\hline Fort Stark & 0.066 & & & & & \\
\hline Quoddy Head & 0.125 & 0.323 & & & & \\
\hline Nubble Light & 0.041 & 0.193 & 0.071 & & & \\
\hline Reid State Park & 0.228 & 0.459 & 0.020 & 0.170 & & \\
\hline Schoodic point & 0.076 & 0.217 & 0.108 & 0.017 & 0.227 & \\
\hline Wiscasset & 0.313 & 0.544 & 0.231 & 0.341 & 0.206 & 0.557 \\
\hline
\end{tabular}

in fitness among lineages) and selection (which decreases the variation in fitness among lineages; Desai and Fisher 2007, Desai et al. 2007, Lang et al. 2011). The result is high levels of genetic variation and considerable variation in fitness within asexual populations.

The marine red alga $P$. umbilicalis does not appear to have a very high mutation rate to allow new lineages to accumulate rapidly, but the passage of time has clearly allowed a number of lineages to accumulate within populations in the Gulf of Maine. We looked at eight EST-SSR loci and found a few polymorphic loci with very few alleles, amounting to six genotypes among seven populations. The numbers of alleles we found at each locus are low compared to most plants (Gupta et al. 1996, Varshney et al. 2005), suggesting that the mutation rate is generally low compared to higher plants. More work on this aspect of $P$. umbilicalis genome biology is necessary before strong conclusions can be drawn, however.
The relatively low number of loci we assayed is sufficient to differentiate large-scale patterns among the populations. More loci may reveal more differences within the genotypes we describe, but is unlikely to change the general pattern of differentiation among genotypes and populations because the genotypes cannot exchange genetic material via sexual reproduction.

\section{Population structure}

Our study considered two populations collected from estuarine tidal rapid sites (Dover Point and Wiscasset), and five populations from open-coastal sites (Fort Stark, Quoddy Head, Schoodic Point, Reid State Park, and Nubble Light). The estuarine tidal rapid sites were very similar to each other; individuals from the estuarine tidal rapid sites were collected from bridge pilings in the high 
intertidal zone where few other organisms were found, presumably because larger organisms would be damaged by the currents produced by tidal flow. The open-coastal sites were more diverse, and individuals were collected from a number of different rock types, exposures, and in areas that experienced considerable variation in wave action and force.

Despite the habitat similarity of the Dover Point and Wiscasset locations, the population from Dover Point shared its lowest genetic distance (i.e. its highest genetic similarity) with the neighboring open-coastal populations at Fort Stark $(d=0.066)$ and Nubble Light $(d=0.041)$. The population at Wiscasset shared the lowest genetic distances with its closest sampled open-coastal population at Reid State Park $(d=0.206)$. The population from Wiscasset was very divergent from other populations, with genetic distances ranging from 0.206 with its closest open-coastal population at Reid State Park to 0.557 with the population at Schoodic Point (Table 4). The genetic differences are largely driven by a high frequency of genotype 5 in the Wiscasset population, which is relatively rare in other populations (Figure 1). Such a high frequency of an otherwise rare genotype could suggest the possibility of a bottleneck during colonization of the bridge pilings at Wiscasset, and the presence of this genotype at the closest open-coastal population at Reid State Park could suggest the two populations share a source population.

Genotype 5 was also present in populations from Fort Stark and Nubble Light, but was not present at Dover Point, and therefore probably does not represent a genotype that is particularly well adapted to estuarine habitats. More importantly, however, the high genetic distances between Dover Point and Wiscasset and the divergent genotype frequencies suggest that isolation-by-distance is more important to population structure than selection within habitat. The estuarine tidal-rapid populations at Dover Point and Wiscasset are more closely related to their closest opencoastal population than they are to each other.

Despite this, isolation-by-distance is not the dominant force shaping population structure among all populations in the Gulf of Maine. A Mantel test among all populations found no correlation between Nei's unbiased genetic distances and geographic distances (Mantel test: $\left.R^{2}=-0.10, p=0.60\right)$. Analysis of Molecular Variance (AMOVA; Excoffier et al. 1992) partitions the variation in allele frequencies within and among populations, and more variation among populations than within populations might suggest genetic isolation and differentiation due to genetic drift or selection. For P. umbilicalis populations in the Gulf of Maine, however, AMOVA revealed that variation was partitioned almost equally within and among populations; slightly more of the variation was within populations (58\%), though a considerable proportion was partitioned among populations $\left(42 \%, \Phi_{\mathrm{PT}}=0.42\right.$, $\mathrm{p}<0.001)$. There is thus no evidence for habitat selection or genetic isolation by distance using these genetic markers.

Lacking direct evidence of habitat selection and isolation-by-distance among all populations, the population structure of $P$. umbilicalis in the Gulf of Maine may be dictated largely by stochastic forces, such as bottlenecks (as for Wiscasset) and genetic drift. Isolation-by-distance appears to be an important factor dictating population structure of estuarine populations, but not an important factor dictating population structure within the Gulf of Maine as a whole. This does not mean that selection for habitat does not occur. The lack of recombination among genotypes means that adaptation in one population may occur along a different trajectory from that in a population in a similar habitat, rather than in parallel with that population. Putatively neutral genetic markers such as these are probably poor markers for identifying population differentiation in asexual populations, because there is no guarantee that the genomes of individuals containing the same EST-SSR are identical. Based on these data presented here, it appears the initial distribution of these genotypes was stochastic, with the exception of estuarine populations, which were probably founded from nearby open-coastal populations after the initial distribution of genotypes. Subsequent diversification from the initial stochastically-dispersed lineages could have occurred in each population, and without sampling on a more genomic scale, there is no way to know if habitat selection shapes population structure in the Gulf of Maine.

\section{New Hampshire lineages}

The most genetic diversity at EST-SSR loci within populations was found at Fort Stark and Dover Point, New Hampshire. The population at Dover Point, however, was sampled more often than the other populations, which could suggest that there is a temporal effect on diversity. In addition to being the most genotypically diverse, populations from New Hampshire were more closely related to each other and to populations in southern Maine than to populations from northern Maine based on Nei's unbiased genetic distances calculated using genotype frequencies (Table 4, Figure 3). The shared genotypes (particularly genotype 3) among them suggest that Fort Stark or other nearby southern Maine populations such as Nubble Light, 
rather than distant populations from Maine, provide some of the genetic material that the population at Dover Point is derived from.

Genotypes 3 at Dover Point and Fort Stark, and genotype 6 at Fort Stark may represent more recent mutations than the other genotypes sampled in this study. They differ from genotype 1 by one allele at different loci, and neither genotype is found in populations from Maine. The degree of similarity among genotypes 1, 3 and 6 suggests the genotypes are related (Figure 2), and the widespread geographic distribution of genotype 1 and its presence among the group of putative recombinant genotypes (discussed below, shown in Table 3) suggest that genotype 1 is probably the ancestral genotype from which genotypes 3 and 6 are derived. Genotype 3 and 6 thus represent lineages created by more recent mutations than those found in other genotypes. If we assume a step-wise migration from the NE Atlantic Ocean via Iceland and maritime Canada in the post-glacial era, as Ingolfsson (1992) does for rocky shore invertebrates, then Fort Stark as the southernmost population may represent the most derived population of Porphyra umbilicalis in this study. The population may represent an accumulation of various genotypes that evolved in more northerly locations and moved south with the prevailing current in the Gulf of Maine. Further studies using controlled seasonal collections on more southern populations would be necessary to test this theory, however, as other modes of transport across the Atlantic were possible (Brawley et al. 2009).

Genotype 6 is the only genotype unique to a single population and is found only at Fort Stark (Figure 1). The genotype was highly reproducible in replicate collections (data not shown), individuals possessing the genotype had $100 \%$ identity to voucher P. umbilicalis samples within the sequenced region of $r b c \mathrm{~L}-r b c \mathrm{~S}$ intergenic spacer, and the genotype could be re-sampled from a precise location in the field. Individuals of that genotype appear to be limited to a single rock on which individuals experience considerable turbulence of low velocity due to the position of that rock in the surf. Individuals tend to have shorter thalli and are of a lighter color. By contrast, genotype 3 was very common at Fort Stark in other locations along the shoreline, and fairly common at Dover Point. The number of samples at Dover Point is small due to the small size of the population, and subsequent collecting trips to the Dover Point site to augment the collection have been difficult due to construction at the U.S.-4/NH-16 bridge (www. newington-dover.com). Our most recent trips have not found any $P$. umbilicalis individuals. Indeed, the transect sites in West et al. (2005) have apparently been covered by the pilings for the new bridge.

\section{Putative recombinant genotypes?}

Four common genotypes (genotypes 1, 2, 4, 5; Table 3) were the product of either convergent evolution of alleles (homoplasy) or a recombination event. The mode of asexual reproduction by mitotic differentiation of blade cells means that there is no recombination of genetic material in populations of Porphyra umbilicalis in the NW Atlantic, and therefore linkage among loci should be complete, regardless of their location on the chromosomes. The linkage among loci 2179 and 2219 is not complete, because alleles A and B from locus 2179 were found with either allele A or B from locus 2219. The lack of complete linkage among these loci suggests that some alleles arose independently in different lineages through convergent evolution, or that a recombination event disrupted the linkage between these two loci. Mitotic recombination could not explain these data because only the haploid life history stage of this species is present in the NW Atlantic, and thus a mitotic recombination event would be undetectable unless it involved unequal crossing over.

The most parsimonious explanation for these data is a recombination event, which casts doubt on the assumption that these populations reproduce exclusively asexually in the NW Atlantic. However, if a local recombination event created these genotypes, we might expect to find all four possible recombinants in a single population. All four putative recombinant genotypes were sampled, but all four were not sampled in a single population. A limited number of populations and individuals from each population were sampled, however, and the missing recombinant genotype from each population could easily have been overlooked by chance.

While these putative recombinant genotypes appear to be the strongest evidence thus far that sexual reproduction in the NW Atlantic could exist, researchers have looked for evidence of sexual reproduction in the NW Atlantic in the past and come up lacking (Blouin and Brawley 2012). In light of that research, a number of possible explanations exist to explain the data presented here without invoking sexual recombination in the NW Atlantic. Our data could be explained by convergent evolution of microsatellite lengths (Figure 4, Scenario 1), which has been found to exist in other organisms (Garza and Freimer 1996, Ortí et al. 1997). Alternatively, the putative recombinant genotypes could represent genotypes resulting from a historic recombination event that took place before the ability to reproduce sexually was apparently lost (i.e. before colonization of the NW Atlantic, Figure 4, Scenario 3) and tissue carrying those genotypes could have subsequently migrated to the NW Atlantic. Given the ambiguity of the 
origin of these genotypes and previous evidence to the contrary (Blouin and Brawley 2012), it is not possible at this time to claim definitively that sexual reproduction occurs in NW Atlantic populations of $P$. umbilicalis, nor is it possible to differentiate between these alternative theories that do not invoke recombination in the NW Atlantic.

\section{How many introductions?}

Populations of Porphyra umbilicalis found on NE Atlantic shores reproduce both sexually and asexually (Brodie and Irvine 2003), but NW Atlantic populations are not known to reproduce sexually (Blouin et al. 2007, Blouin 2010, Blouin and Brawley 2012). If NW Atlantic populations are derived from NE Atlantic populations (Teasdale and Klein 2010), it is not clear why NW Atlantic populations are apparently incapable of reproducing sexually (though to our knowledge, no one has studied ecological differences in sexual and asexual populations in the NE Atlantic that may prevent sexual populations from becoming established in the NW Atlantic). Efforts have been made to detect the signatures of sexual reproduction and there is no current evidence that sexual reproduction occurs (Blouin and Brawley 2012).

However, an asexual mode of reproduction would have been highly advantageous for $P$. umbilicalis as it colonized the NW Atlantic from European refugia (Figure 4, Scenario 1). The advantage of asexual reproduction or other forms of self-compatibility in colonizing species has long been known to invasive species biology (Baker and Stebbins 1965). Organisms that are capable of reproducing asexually are considered more likely to be successful in colonizing new habitats than species that require an alternative mating type. For species with alternative mating types to be successful in colonizing new habitats, the colonization event by necessity must include sufficient numbers of both mating types to ensure reproduction. The statement has been codified as "Baker's Law" (Baker 1967, Cheptou 2011), and support has been found in a number

\section{Single Introduction NW Atlantic NE Atlantic}

Scenario 1:

Asexual Population Sexual Population

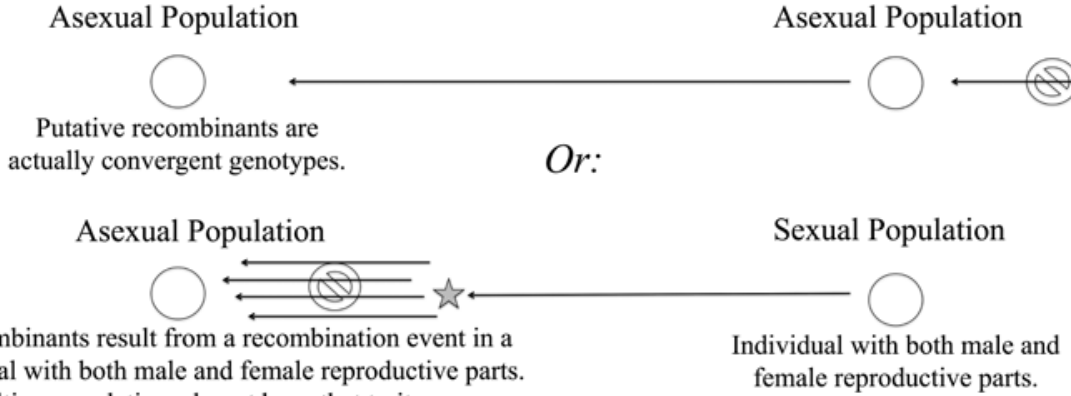

single individual with both male and female reproductive parts.

Resulting populations do not have that trait.

female reproductive parts.

\section{Multiple Introductions}

Scenario 3:

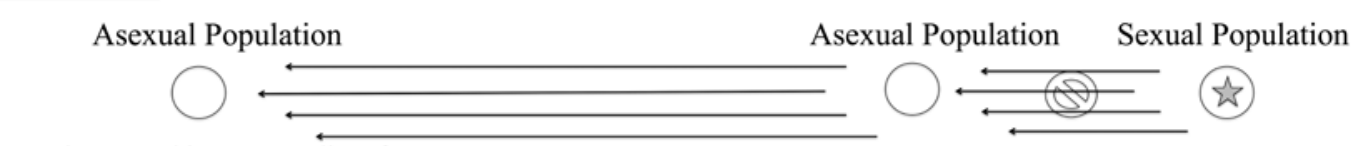

Putative recombinants are relics of an ancestral recombination event.

Scenario 4:

But then, why not?

Asexual Population Unidentified Sexual Population(s)?

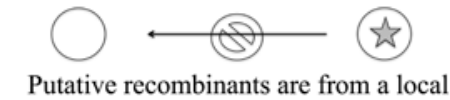

Putative recombinants are from a local unidentified or extirpated sexual population.

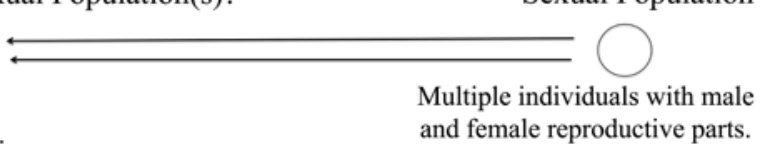

Sexual Population and female reproductive parts.
Recombination event that created putative recombinants.

Sexual reproduction (i.e. recombination) is lost.

Figure 4: Four possible scenarios that explain colonization of the NW Atlantic from the NE Atlantic that attempt to reconcile data from this study and others that find no sexual reproduction in the NW Atlantic. 
of noxious, terrestrial invasive species (Hollingsworth and Bailey 2000, Hao et al. 2011). Most P. umbilicalis individuals are dioecious when sexually reproductive (Brodie and Irvine 2003, Brodie et al. 2008), and thus would have required the introduction of multiple mating types to sustain a sexual population. Some individuals contain both male and female reproductive structures (Holmes and Brodie 2004), but it is not clear precisely how common this trait is in $P$. umbilicalis, or how heritable that trait is. Holmes and Brodie (2004) found that only 5\% of Porphyra dioica individuals in Devon, U.K., which is usually dioecious like $P$. umbilicalis, contained both male and female reproductive parts, and $P$. umbilicalis individuals apparently with both male and female reproductive tissue in the Faroe Islands are probably equally rare (personal communication, Agnes Mols-Mortensen). In addition, if a rare individual containing both male and female reproductive structures were to colonize NW Atlantic shores, it is not clear what percentage, if any, of subsequent generations would be similar. Male reproductive structures are apparently absent from NW Atlantic populations of P. umbilicalis at this time (Bird and McLachlan 1992). The ability to reproduce asexually via neutral spores would have provided the initial colonist (whether that colonist was an asexual individual as in Figure 4 Scenario 1, or an individual with both male and female reproductive parts as in Figure 4 Scenario 2) the ability to establish a population and persist following a single introduction.

Support for Baker's Law is somewhat limited (Rambuda and Johnson 2004, Grimsby et al. 2007, Barrett et al. 2008), however, because multiple introductions appear to be quite common in terrestrial plants (reviewed in Dlugosch and Parker 2008) and marine colonizers/ invaders (Dlugosch and Parker 2008, Brawley et al. 2009), thereby eliminating the advantage of clonal invasives. Indeed, there is evidence that the colonization event of $P$. umbilicalis did in fact involve multiple individuals. If we assume that NW Atlantic populations are incapable of sexual reproduction, then it is possible the putative recombinant genotypes identified in the present study represent a recombination event that occurred prior to the colonization of NW Atlantic shores. If these putative recombinants are relics of ancestral, sexual populations in Europe, then multiple introductions from refugia in Europe would have occurred for all four putative recombinant genotypes to occur in the NW Atlantic (Figure 4 Scenario 3).

Therefore the answer at this time to the question regarding how many introductions of $P$. umbilicalis from the NE Atlantic to the NW Atlantic occurred remains unclear. Teasdale and Klein (2010) suggested there was just one introduction of material from European refugia, but their markers were relatively conserved ribosomal intron and internal transcribed spacers, which could have missed cryptic genetic diversity. Given that P. umbilicalis is capable of asexual reproduction, a single introduction could have established the populations in the NW Atlantic, and asexual reproduction would have been an advantage to colonizers (Figure 4 Scenario 1 and 2). However, our data suggest that multiple introductions from the NE Atlantic could have occurred (i.e. all four putative recombinant genotypes can be found in the Gulf of Maine; Figure 4 Scenario 3). If we allow for the possibility of multiple introductions, however, it begs the question of why individuals capable of sexual reproduction were not included, and why we do not find sexual populations in the Gulf of Maine that were themselves capable of producing the recombinant genotypes (Figure 4 Scenario 4). The introduction of a single individual containing both male and female reproductive parts seems to explain all aspects of the data (Figure 4 Scenario 2), but the apparent rarity of these individuals in the $\mathrm{NE}$ Atlantic makes this possibility seem unlikely.

\section{Conclusions}

Far from being clonal throughout the Gulf of Maine, populations of Porphyra umbilicalis exhibit genetic diversity within and among populations. High genotypic diversity and variation in fitness within populations are predicted by theoretical models of evolution in asexual populations. Population substructure in the Gulf of Maine is probably dictated largely by stochastic forces, such as bottlenecks and genetic drift, rather than clustering of genotypes due to adaptation for similar atypical habitats.

Acknowledgments: Samples at Schoodic Point, Acadia National Park were collected under permit number \#ACAD-2012-SCI-0013 issued to Dr. Jessica Muhlin from April 16, 2012, to April 25, 2012. We thank Dr. Agnes MolsMortensen, Dr. Chris Benton, Yuanyu Cao, Greg Smith, Anna, and Sean and Darwin Harrison for help in collections. Dr. Chris Neefus provided culture room space, statistical advice, and valuable feedback. Dr. Andrew Price and Shay Polosky provided the Thermo Scientific NanoDrop 2000c and technical advice. Many thanks also to Jobriah Anderson at the Hubbard Center for Genomic Studies who conducted the genotyping. Partial funding was provided by the New Hampshire Agricultural Experiment Station. This is Scientific Contribution Number 2584. This work was supported by the USDA National Institute of Food and Agriculture Hatch Project 0222799. 


\section{References}

Baker, H.G. 1967. Support for Baker's law-as a rule. Evol. 21: 853-856.

Baker, H.G. and G. Stebbins. 1965. Characteristics and modes of origin of weeds. In: (H. G. Baker and G. Stebbins eds.) The genetics of colonizing species. Academic Press, New York, pp. 147-168.

Barrett, S.C., R.I. Colautti and C.G. Eckert. 2008. Plant reproductive systems and evolution during biological invasion. Mol. Ecol. 17: 373-383.

Bird, C.J., J.L. McLachlan. 1992. Seaweed flora of the maritimes: Rhodophyta - the red algae. Biopress, New York, NY. $177 \mathrm{pp}$.

Blouin, N. 2010. Asexual reproduction in Porphyra umbilicalis Kützing (Rhodophyta) and its development for use in mariculture. University of Maine, School of Marine Sciences, Orono, ME. $150 \mathrm{pp}$.

Blouin, N.A. and S.H. Brawley. 2012. An AFLP-based test of clonality in widespread, putatively asexual populations of Porphyra umbilicalis (Rhodophyta) in the Northwest Atlantic with an in silico analysis for bacterial contamination. Mar. Biol. 159: 2723-2729.

Blouin, N., F. Xiugeng, J. Peng, C. Yarish and S.H. Brawley. 2007. Seeding nets with neutral spores of the red alga Porphyra umbilicalis (L.) Kützing for use in integrated multi-trophic aquaculture (IMTA). Aquaculture 270: 77-91.

Blouin, N.A., J.A. Brodie, A.C. Grossman, P. Xu and S.H. Brawley. 2011. Porphyra: a marine crop shaped by stress. Trends Plant Sci. 16: 29-37.

Brawley, S.H., J.A. Coyer, A.M.H. Blakeslee, G. Hoarau, L.E. Johnson, J.E. Byers, W.T. Stam and J.L. Olsen. 2009. Historical invasions of the intertidal zone of Atlantic North America associated with distinctive patterns of trade and emigration. PNAS 106: 8239-8244.

Bray, T.L., C.D. Neefus and A.C. Mathieson. 2006. Morphological and molecular variability of Porphyra purpurea (Roth) C. Agardh (Rhodophyta, Bangiales) from the Northwest Atlantic. Nova Hedwigia 82: 1-22.

Brodie, J.A. and L.M. Irvine. 2003. Seaweeds of the British Isles Vol. 1 Rhodophyta Part 3B Bangiophycidae. The Natural History Museum, London, London. pp. 130-132.

Brodie, J., L.M. Irvine, C.D. Neefus and S. Russell. 2008. Ulva umbilicalis L. and Porphyra umbilicalis Kütz. (Rhodophyta, Bangiaceae): a molecular and morphological redescription of the species, with a typification update. Taxon 57: 1328-1331.

Chakraborty, R., M. Kimmel, D.N. Stivers, L.J. Davison and R. Deka. 1997. Relative mutation rates at di-, tri-, and tetranucleotide microsatellite loci. PNAS 94: 1041-1046.

Chan, C.X., N.A. Blouin, Y. Zhuang, S. Zäuner, S.E. Prochnik, E. Lindquist, S. Lin, C. Benning, M. Lohr, C. Yarish, E. Gantt, A.C. Grossman, S. Lu, K. Müller, J.W. Stiller, S.H. Brawley, and D. Bhattacharya. 2012. Porphyra (Bangiophyceae) transcriptomes provide insights into red algal development and metabolism. J. Phycol. 48: 1328-1342.

Cheptou, P.-0. 2011. Clarifying Baker's law. Ann. Bot-London 109: 633-641.

Chessel, D., A.B. Dufour and J. Thioulouse. 2004. The ade4 package - One-table methods. R News 4: 5-10.
Desai, M.M. and D.S. Fisher. 2007. Beneficial mutation-selection balance and the effect of linkage on positive selection. Genetics. 176: 1759-1798.

Desai, M.M. and D.S. Fisher. 2011. The balance between mutators and nonmutators in asexual populations. Genetics. 188: 997-1014.

Desai, M.M., D.S. Fisher and A.W. Murray. 2007. The speed of evolution and maintenance of variation in asexual populations. Curr. Biol. 17: 385-394.

Dlugosch, K.M. and I.M. Parker. 2008. Founding events in species invasions: genetic variation, adaptive evolution, and the role of multiple introductions. Mol. Ecol. 17: 431-449.

Dray, S. and A.B. Dufour. 2007. The ade4 package: implementing the duality diagram for ecologists. J. Stat. Softw. 22: 1-20.

Dray, S., A.B. Dufour and D. Chessel. 2007: The ade4 package-II: Two-table and K-table methods. R News. 7: 47-52.

Excoffier L. and H.E.L. Lischer. 2010. Arlequin suite ver 3.5: a new series of programs to perform population genetics analyses under Linux and Windows. Mol. Ecol. Res. 10: 564-567.

Excoffier, L., P.E. Smouse and J.M. Quattro. 1992. Analysis of molecular variance inferred from metric distances among DNA haplotypes-application to human mitochondrial-DNA restriction data. Genetics. 131: 479-491.

Excoffier L., G. Laval, S. Schneider. 2005. Arlequin (version 3.0): an integrated software package for population genetics data analysis. Evol. Bioinform. 1: 47-50.

Garza, J.C. and N.B. Freimer. 1996. Homoplasy for size at microsatellite loci in humans and chimpanzees. Genome Res. 6: 211-217.

Good, B.H., I.M. Rouzine, D.J. Balick, O. Hallatschek and M.M. Desai. 2012. Distribution of fixed beneficial mutations and the rate of adaptation in asexual populations. P. Natl. Acad. Sci USA 109: 4950-4955.

Grimsby, J.L., D. Tsirelson, M.A. Gammon and R. Kesseli. 2007. Genetic diversity and clonal vs. sexual reproduction in Fallopia spp. (Polygonaceae). Am. J. Bot. 94: 957-964.

Gupta, P.K., H.S. Balyan, P.C. Sharma, B. Ramesh. 1996. Microsatellites in plants: a new class of molecular markers. Curr. Sci. 70: 45-54.

Hao, J.H., S. Qiang, T. Chrobock, M. van Kleunen, Q.Q. Liu. 2011. A test of Baker's law: breeding systems of invasive species of Asteraceae in China. Biol. Inv. 13: 571-580.

Hollingsworth, M.L. and J.P. Bailey. 2000. Evidence for massive clonal growth in the invasive weed Fallopia japonica (Japanese knotweed). Bot. J. Linn. Soc. 133: 463-472.

Holmes, M.J., J. Brodie. 2004. Morphology, seasonal phenology and observations on some aspects of the life history in culture of Porphyra dioica (Bangiales, Rhodophyta) from Devon, UK. Phycologia 43: 176-188.

Ingolfsson, A. 1992. The origin of the rocky shore fauna of Iceland and the Canadian maritimes. J. Biogeogr. 19: 705-712.

Kryazhimskiy, S., D.P. Rice and M.M. Desai. 2012. Population subdivision and adaptation in asexual populations of Saccharomyces cerevisae. Evolution 66: 1931-1941.

Lang, G.I., D. Botstein and M.M. Desai. 2011. Genetic variation and the fate of beneficial mutations in asexual populations. Genetics 188: 647-661.

Mathieson, A.C., E.J. Hehre, N.B. Reynolds. 1981. Investigations of New England marine algae II: the species compositions, distribution and zonation of seaweeds in the great bay estuary system and the adjacent open coast of new hampshire. Bot. Mar. 24: 533-546. 
Mols-Mortensen, A., C.D. Neefus, R. Nielsen, K. Gunnarsson, S. Egilsdóttir, P.M. Pedersen and J. Brodie. 2012. New insights into the biodiversity and generic relationships of foliose Bangiales (Rhodophyta) in Iceland and the Faroe Islands. Eur. J. Phycol. 47: 146-159.

Nelson, W.A., J. Brodie and M.D. Guiry. 1999. Terminology used to describe reproduction and life history stages in the genus Porphyra (Bangiales, Rhodophyta). J. Appl. Phycol. 11: 407-410.

Orti, G., D.E. Pearse and J.C. Avise. 1997. Phylogenetic assessment of length variation at a microsatellite locus. PNAS 94: 10745-10749.

Page, A.F. 2010. Application note: detection and avoidance of polysaccharides in plant nucleic acid extractions thermo fisher scientific, Wilmington, DE. http://www.thermo.com.cn/ Resources/201305/3110520421.pdf.

Peakall, R. and P.E. Smouse. 2006. GenAlEx 6: genetic analysis in Excel. Population genetic software for teaching and research. Mol. Ecol. Notes 6: 288-295.

Peakall, R. and P.E. Smouse. 2012. GenAlEx 6.5: genetic analysis in Excel. Population genetic software for teaching and researchan update. Bioinformatics 28: 2537-2539.

R Core Team (2015). R: a language and environment for statistical computing. R Foundation for Statistical Computing, Vienna, Austria. ISBN 3-900051-07-0, URL http://www.R-project.org/.

Rambuda, T.D. and S.D. Johnson. 2004. Breeding systems of invasive alien plants in South Africa: does Baker's rule apply? Divers. Distrib. 10: 409-416.

Rozen, S. and H. Skaletsky. 2000. Primer3 on the WWW for general users and for biologists programmers. In: (S. Misener and S.A. Krawetz, eds.) Bioinformatics methods and protocols. Humana Press, Totowa, NJ. pp. 365-386.

Smith, D.R., J. Hua, R.W. Lee and P.J. Keeling. 2012. Relative rates of evolution among the three genetic compartments of the red alga Porphyra differ from those of green plants and do not correlate with genome architecture. Mol. Phylogenet. Evol. 65: 339-344.

Teasdale, B.W. and A.S. Klein. 2010. Genetic variation and biogeographical boundaries within the red alga Porphyra umbilicalis (Bangiales, Rhodophyta). Bot. Mar. 53: 417-431.

Teasdale, B., A. West, H. Taylor and A. Klein. 2002. A simple restriction fragment length polymorphism (RFLP) assay to discriminate common Porphyra (Bangiophyceae, Rhodophyta) taxa from the Northwest Atlantic. J. Appl. Phycol. 14: 293-298.

Thiel, T., W. Michalek, R.K. Varshney and A. Graner. 2003. Exploiting EST databases for the development and characterization of gene-derived SSR-markers in barley (Hordeum vulgare L.). Theor. Appl. Genetics 106: 411-422.

Thompson, D.A., M.M. Desai and A.W. Murray. 2006. Ploidy controls the success of mutators and nature of mutations during budding yeast evolution. Curr. Biol. 16: 1581-1590.

Varshney, R.K., A. Graner, M.E. Sorrells. 2005. Genic microsatellite markers in plants: features and applications. Tr. Biotech. 23: 48-55.

Weissman, D.B., M.M. Desai, D.S. Fisher and M.W. Feldman. 2009. The rate at which asexual populations cross fitness valleys. Theor. Pop. Biol. 75: 286-300.
West, A.L., A.C. Mathieson, A.S. Klein, C.D. Neefus and T.L. Bray. 2005. Molecular ecological studies of New England species of Porphyra (Rhodophyta, Bangiales). Nova Hedwigia 80: 1-24.

\section{Bionotes}

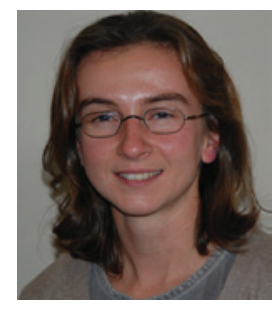

Renée L. Eriksen
Department of Biological Sciences,
University of New Hampshire, Durham, NH
03824, USA; and United States Department
of Agriculture - Agricultural Research
Service, 1636 East Alisal Street, Salinas, CA
93905, USA
renee.eriksen@gmail.com

Renée L. Eriksen earned her PhD in Plant Biology from the University of New Hampshire in 2014 for her work on the population genetics and stress physiology of the marine red alga Porphyra umbilicalis. She has previously worked on the population genetics of lungless salamanders and of invasive weeds. She is currently a postdoctoral researcher for the Agricultural Research Service, United States Department of Agriculture in Salinas, CA, USA and her current work focuses on understanding stress physiology and gene expression in lettuce during drought.

\section{Lindsay A. Green}

Department of Biological Sciences, University of New Hampshire, Durham, NH 03824, USA; and Department of Biological Sciences, University of Rhode Island, Kingston, RI 02881, USA

Lindsay A. Green is currently a postdoctoral researcher at the University of Rhode Island. She received her PhD in Plant Biology from the University of New Hampshire in 2014 for work on the physiology of Northwest Atlantic nori (Porphyra and Pyropia) with the goal of developing new crops for aquaculture in the United States. She has also conducted research on introduced seaweeds and kelp forest ecology. More recently, her postdoctoral research has focused on the ecology of bloom-forming macroalgae (i.e. Ulva spp.).

\section{Anita S. Klein}

Department of Biological Sciences, University of New Hampshire, Durham, NH 03824, USA

Anita S. Klein is an Associate Professor of Biology at the University of New Hampshire. Her PhD is in Biochemistry from Michigan State University and she did postdoctoral studies in the Laboratory of Genetics at the University of Wisconsin. Twenty years ago, she brought molecular evolution and population genetic approaches to a survey of Porphyra in the Gulf of Maine. Most recently, she has been exploring genetic diversity in Porphyra umbilicalis populations for nutrient uptake and thermal tolerance that might be exploited to improve integrated multitrophic aquaculture systems. Anita has a half-time administrative appointment in the New Hampshire Agricultural Experiment Station. 